\title{
LE SIGNE AGISSANT. D'UNE SÉMIOLOGIE \\ DE LA MIMESIS VERS UNE PRAGMATIQUE \\ DE LA PERFORMANCE
}

\section{THE SIGN ACTING. FROM SEMIOLOGY OF MIMESIS TO A PRAGMATICS OF PERFORMANCE}

\author{
Nicoleta POPA BLANARIU \\ Université Vasile Alecsandri (Rumanía) \\ npopablanariu@yahoo.com
}

\begin{abstract}
Resumen: Muchos experimentos artísticos en la performance contemporánea se centran en lo orquestal (Escuela de Palo Alto), en los aspectos más bien no intencionales de comunicación: los co-particpantes y los co-creadores del espectáculo a menudo participan involuntariamente debido a la (auto)comunicación. Desde el punto de vista de la observación de la audiencia, los actos ilocucionarios que se realizan en el escenario, en una actuación teatral tradicional, son actos reales dentro de un universo de ficción (Searle). Pero en realidad sucede algo muy diferente en el drama ritual, desde el punto de vista de los participantes, ya que los actos reales se llevan a cabo dentro de un mundo real. El drama ritual tiene este valor altamente performativo, por lo que es un medio de actuar sobre lo real.
\end{abstract}

Palabras clave: Mímesis. Semiótica. Pragmática. Performance. Ritual.

Abstract: Many artistic experiments in the contemporary performance are focused on the orchestral (Palo Alto School), rather non-intentional aspects of communication: the audience co-participates and co-creates the show, often (sel-)communicating unintentionally From the viewpoint of the observing audience, the illocutionary acts performed on stage, in a traditional theatrical performance, are real acts within a fictional universe (Searle). But it's really quite different in the ritual drama, where from the participants viewpoint, real acts are being performed within a real world. The ritual drama has this highly performative value, it is a means of acting on the real.

Key Words: Mimesis. Semiotics. Pragmatics. Performance. Ritual. 


\section{FONDEMENTS D’UNE SÉMIOLOGIE DES ARTS DU SPECTACLE: LUCIEN DE SAMOSATE ET AUGUSTIN}

À deux siècles d'intervalle, Lucien de Samosate (2009; 1983) et Augustin (2002) entament une réflexion théorique sur la danse. Leurs points de vue doivent beaucoup aux époques où ils ont vécu, et vacillent entre la sémiotique, une (toute première) esthétique de la réception et, surtout chez Augustin, la morale. Augustin reste partagé dans son jugement des arts, et sur ce point, il s'éloigne de son devancier Lucien. Celui-ci suit la danse sans parti pris; ni ses remarques, ni ses conseils n'obéissent à telle ou telle doctrine. Tandis que la sémio-esthétique de la danse qu'esquisse Augustin, tout comme son aperçu des arts du spectacle -héritage à blâmer, dit-il, des temps païens- se prête à tous les apriorismes et à toutes les réprimandes (Augustin, 2002: 165). Plus précisément, l'évêque d'Hippone range les arts "imitatifs", y compris les arts du spectacle, parmi les "institutions inutiles", voire pires, tant qu'elles portent atteinte à la bonne conduite des citoyens et causent I'“affaiblissement de la vertu" (Augustin, 2002).

Outre l'intention de leurs commentaires (moralisatrice ou pas), ce qui sépare encore Lucien d'Augustin ce sont leurs vues différentes sur l'interprétation du mouvement. À l'opinion de Lucien, les gestes de danse doivent être "clairs" tels qu'on n'ait plus alors besoin d'en aller chercher le sens chez les "connaisseurs". Les mouvements vont mettre au jour le sens des chants qui les accompagnent (Samosate, 1983: 235). Lucien demande la transparence iconique du signe - à son avis, condition sine qua non de la compréhension. Le point de vue d'Augustin (2002) repose sur l'ancienne pratique des spectacles donnés jadis à Carthage, qu'il ne connaissait que par ouï-dire. Ainsi, Augustin évoque la charge herméneutique, métadiscursive que le héraut assumait jadis à Carthage. À cette époquelà, on appelait le héraut à tirer au clair les significations occultées des danses qu'on pouvait voir sur scène (Augustin, 2002:165). Le héraut était donc une instance que le socius avait investie de la construction (ou de la préservation) du sens de la danse. Tel était ce heraut en vertu de la connaissance qu'il avait d'un discours fondateur, auquel on faisait appel lors de toute (re)mise en scène. Une fois apprise la leçon du passé, et l'efficacité de l'ancien héraut une fois confirmée, Augustin n'hésite pas à en réclamer les compétences pour entendre au plus juste les spectacles de son temps. (Demander ainsi un métadiscours du spectacle vaut la légitimation -peut-être pour la première fois- des métiers tels critique et chroniqueur dramatique ou de danse).

Sur certains points, la réflexion d'Augustin sur les signes chorégraphiques annonce les thèses de Peirce, y compris sa fameuse trichotomie: icône, indice, symbole -disait Peirce (1978)- sont, à l'état pur, des catégories à la seule existence théorique. Au cours de leur fonctionnement, ils empruntent l'un de l'autre des caractères spécifiques: dans certaines conditions, une icône ou un indice peut accomplir aussi le rôle du symbole (Peirce, 1978). 
Ce qui revient à une conclusion classique de Saussure (1997; 1998): la motivation (des indices comme des icônes, avec les mots de Peirce) et l'arbitraire (du symbole de Peirce, (re)lié à une convention) sont toujours relatives. La conclusion d'Augustin n'en était pas très loin: généralement, la semiosis-disait-il-s'établit de telle manière que les signes soient pareils à ce qu'ils désignent (Augustin, 2002: 165). Mais la ressemblance -remarquait-ilpeut se manifester sous plusieurs aspects. Par conséquent, il faut arriver à un "consensus" (Augustin, 2002). Ce n'est que cela qui fait possible la transmission ou le partage du sens; sinon, on s'enfonce dans l'idiosyncrasie. Donc avant Saussure, Augustin établissait que ce "consensus" était absolument nécessaire, voire obligatoire. Particulièrement, l'icône même -comme representamen, n'importe quelle soit sa forme d'expression- n'existe qu'en vertu du consensus qui la relie à ce qu'elle désigne. Apparemment tout à fait "naturelle" (Augustin) -ce qui veut dire spontanée, immédiate -, cette relation de l'icône à son objet s'impose en fait par "I'habitude" (Peirce) ou bien par une "convention"(Saussure), donc elle fait l'objet d'un "consensus" (Augustin). Il s'ensuit que même les signes "motivés" (Saussure) -tels l'icône et l'indice, dans une taxonomie à la Peirce-comportent aussi une dimension conventionnelle, autrement dit "symbolique", au sens que Peirce donnait à ce mot. Eux non plus, les signes "motivés" n'arrivent à s'échapper au "conventionnel". Il en témoigne la danse carthaginoise, qu'Augustin trouvait ambigüe, voire obscure. Pour la mise au jour des significations du spectacle, le "héraut" était donc indispensable, on en a déjà parlé. À l'opposée, il y a la danse "claire", dont Lucien de Samosate faisait l'éloge: la danse qui rend et imite des actions et des passions (Samosate, 1983: 236). Mais chez Augustin (2002: 165), les significations se présentent à l'esprit selon les conventions de chaque société. C'est-à-dire, la diversité des (sous)codes sociaux et culturels débouche sur la variabilité contextuelle des significations du spectacle.

\section{SIGNIFICATION ET COMMUNICATION. MODÈLE FONCTIONNEL, MODËLE ORCHESTRAL}

Les remarques d'Augustin, mentionnées ci-dessus, anticipent la dissociation moderne entre la sémiologie de la signification et la sémiologie de la communication. La seconde est toujours intentionnelle-comme laisse entendre Saussure- et repose sur un code que partagent l'émetteur et le destinataire. La première, comme chez Peirce, n'exige qu'un destinataire (intelligent consciousness) à même d'interpréter les signes. Ceux-ci ne sont pas obligatoirement intentionnels, car il n'y a pas toujours quelqu'un qui les produise délibérément. À l'opinion de Eco, c'est juste l'intentionnalité qui permet la dissociation communication/ signification (Eco, 1982: 26-29). Cependant, l'intentionnalité perd sa qualité de marque distinctive dans les années '40, avec le Collège invisible de Palo Alto et avec le paradigme de la Nouvelle Communication qu'assume celui-ci. Dans 
cette acception, la communication s'annexe aussi le domaine de la signification non intentionnelle. Tout processus de communication repose sur un système ou code de signification, remarquait Eco (1982: 19). Pourtant, dans le nouveau paradigme de l'École de Palo Alto, la rigueur de cette dichotomie (signification/ communication) s'affaiblit. Plus précisément, la communication ne s'y rapporte "pas seulement à la transmission des messages verbaux, explicites et intentionnels", mais beaucoup plus largement, elle "inclurait l'ensemble des processus par lesquels les sujets s'influencent mutuellement". Une telle définition repose sur "la prémisse que toute action et tout événement offrent des aspects communicatifs, dès qu'ils sont perçus par un être humain" (Bateson et Ruesch, 1988: 6).

Cette nouvelle acception de la communication, qu'apportent l'anthropologue Bateson et le psychiatre Ruesch, établit (presqu')une identification entre "comportement (en présence d'au moins une seconde personne), message et comunication" (Winkin, 2001: 58-59). Mais cela pose un problème: en fin de compte, "qu'est-ce qui n'est pas de la communication?" (Bateson et Ruesch, 1988: 18). Dans une telle perspective, la "nouvelle" communication (dite "orchestrale") dépend absolument d'une "interaction hic et nunc" (Bateson et Ruesch, 1988) et s'éloigne de ses anciens modèles mathématiques et psychologiques (ou "télégraphiques"). Dans de tels modèles, la communication "reposait sur l'émetteur et son intention d'envoyer un message (à un autre individu)", tandis que, dans la perspective de la Nouvelle Communication qu'ils assument, Ruesch et Bateson "partent du récepteur et sa perception d'impressions' en provenance non seulement d'autres individus mais de lui-même, d'événements, de 'l'environnement "' (Winkin, 2001: 55). On y intègre même un aspect non intentionnel de la communication. Cela veut dire que par sa nature d'"animal social", l'homme est "biologiquement contraint" à communiquer: "Nos organes de sens sont constamment en alerte et enregistrent en permanence les signaux reçus; en retour, les organes ainsi stimulés ne sont jamais au repos et nous envoyons donc continuellement des messages vers le monde extérieur" (Bateson et Ruesch, 1988: 19).

À mon avis, c'est justement cet aspect orchestral et plutôt non intentionnel de la communication que certaines formules expérimentales de la performance postmoderne exploitent notamment; le public y intervient en temps réel et (co)participe, en (se) communiquant même à son insu, ou plus qu'il ne le veule. La performance se joue ainsi dans un entre-deux: entre un certain cadre ou scénario relativement prémédité de l'action, d'une part, et la réaction spontanée, imprévisible du public, d'autre part; le public y devient vraiment un partenaire des dans(act)eurs, et trouve ad hoc son rôle dans un genre d'histoire-action (mi-réelle, mi-fictionnelle), qui se tisse sur-lechamp. Ce changement dans la construction du spectacle entraîne un changement d'optique dans la réception. Dans un spectacle classique, le spectateur regarde tout de 
l'extérieur, sans se mêler à ce qui se passe sur scène, tandis que dans la performance, le spectateur -qui est une "conscience interprétante" (Peirce)- s'immerge souvent dans le système spectaculaire qu'il envisage. Selon l'axiome de la Nouvelle Communication (ou communication orchestrale),"on ne peut ne pas communiquer, tout "communique"-voire même (nous) trahit- dans la relation interpersonnelle la plus habituelle, tout comme dans les circonstances spécifiques d'un spectacle. Hamlet -ou Shakespeare- le savait bien, et bien avant que le Collège invisible le dise dans une théorie de la communication renouvelée. Ce n'est qu'ainsi qu'il est devenu possible le psychodrame auquel Hamlet soumettait Claudius à l'insu de ce dernier.

Dans l'interaction quotidienne, tout comme dans les circonstances d'un spectacle, le geste communique, le corps "parle" plus ou moins consciemment (Sapir, 1967), l'espace transmet des messages à sa façon (Hall, 1971), le rythme (visuel, sonore ou proprioceptif, du battement du coeur ou de la respiration) "transmet" à son tour une idée, exprime ou induit une émotion, laisse deviner un état d'esprit. La musique ou le silence "parle" aussi (Tarasti, 1996). Le spectacle (post)moderne -pas une fois interactif, en ce qui concerne les rapports des artistes avec le public- semble valider également un autre axiome de la Nouvelle Communication: "Un individu ne communique pas, il prend part à une communication dont il devient un élément"; "il n'est pas l'auteur de la communication, il y participe" (Birdwhistell apud Winkin, 1984: 75). On y va revenir.

Les prises de position de Lucien et d'Augustin témoignent de l'existence de deux parcours de la danse, que sa production et son interprétation suivent depuis des siècles. Cette ambivalence file toute I'histoire du spectacle et de son métadiscours: depuis Lucien de Samosate et Saint Augustin jusqu'à la rivalité -bien connue au XXe et au XXle siècles- de la danse "pure" (qui s'échappe à la toute puissance de la mimesis) d'avec la danse théâtrale. Le XVIII e siècle connaît lui aussi cette ancienne querelle. À cette époquelà, une danse essentiellement technique et ludique, qui faisait simplement preuve de grâce et d'adresse, passait pour un art superficiel. Et cela, parce que son but unique était de produire des effets pour le plaisir du spectateur. Seule était alors digne d'estime la danse à même de rendre les traits spécifiques de l'individu et ses états d'esprit à travers le mime. Ce qui revient à la danse théâtrale, donc au triomphe absolu de l'iconicité et de la mimesis. Réitéré par Noverre, ce principe qui s'empare de la danse à l'époque des Lumières est celui de l'expressivité émotionnelle et de la logique narrative des mouvements. La concurrence de ces deux genres ne manque pas au XX e et XXI e siècles. Ainsi, à l'opposée du "ballet abstrait", que Ballanchine illustre brillamment, la danse expressionniste est "motivée" en vertu de sa liaison avec un contenu psychologique. Le ballet classique, avec ses caractères en action, est à son tour psychologiquement motivé et pourvu aussi d'une cohérence narrative. 
On peut décrire cette ambivalence -danse pure/théâtrale ou bien, pure/mimiquepar recours au modèle fonctionnel de Jakobson (1963). Dans la danse pure, la fonction dominante est métalinguistique (la fonction du code), qui épouse souvent la fonction poétique, "accent mis sur le message pour son propre compte" (Jakobson, 1963: 218). Dans la danse théâtrale, c'est la fonction référentielle qui assujettit la structure sémantique du message, car ce qui compte, en tout premier lieu, c'est l'objet du discours (designatum), et moins son sujet. Dans la danse classique, il y a la fonction référentielle et aussi la fonction poétique qui prédominent. Dans les ballets romantiques, la fonction expressive (émotive) est assez importante; grâce à elle, l'artiste peut laisser s'entrevoir son univers intérieur, distinct du personnage qu'il interprète sur scène, mais mis au service de celui-ci. D'autres fonctions n'y jouent qu'un "rôle subsidiaire, accessoire" (Jakobson, 1963). La prédominance de la fonction poétique dans le ballet classique est renforcée par la codification stricte des formes d'expression chorégraphique, limitées à un inventaire (ou vocabulaire kinésique) bien défini. Fondée sur l'innovation et sur le mouvement personnalisé, la danse (post)moderne est centrée sur les fonctions: expressive (qui s'organise autour de l'émetteur), phatique (concernant la solidarité intersubjective des danseurs entre eux-mêmes et de ceux-ci avec le public), et conative (Popa Blanariu, 2013a; 2015). Quant à la conative, il est à remarquer que certaines formules du spectacle contemporain, influencées par les techniques du happening, inaugurées dans les années 1950-1960, en Amérique, tentent d'emporter le public en tant que participant actif dans l'espace de jeu. En quelque mesure, le ballet politique-qui s'est emparé de la Cour de France, au XVI ième siècle-est ainsi remplacé par happening et agit-prop. Depuis toujours, la dimension esthétique de l'art est en concurrence avec une autre, plus pratique -sociopolitique et magique-rituelle y comprises (Popa Blanariu, 2013b; Popa Blanariu, 2015). II en témoigne aussi la fonction de la danse (guerrière ou pas) dans l'ancienne cité grecque (Platon, Lois), ou le mélange de mystique, art et politique connu sous le nom de Thingspiel, développé pendant la propagande du Führer en Allemagne.

\section{3. (RE)PRÉSENTATION: MIMESIS VS. PERFORMANCE}

Depuis la deuxième moitié du XXième siècle, la performance, produit symptomatique du postmodernisme, fait l'objet de bien des débats, en Europe et aux États-Unis, et polarise la création artistique, voire plus. Erwing Goffman (2001) étudie "la mise en scène de la vie quotidienne". Richard Schechner assimile tous les comportements sociaux organisés à la performance; à son opinion, "n'importe quelle action encadrée, offerte, soulignée ou exposée est une performance" (Schechner apud Pavis, 2007: 17). André Helbo (2011) souligne les "processus performatifs complexes" qui s'attaquent à la frontière entre vie et spectacle. Patrice Pavis (2007: 16-17) veut "éviter de parler à 
tout bout de champ de 'théâtre', risquant alors de plaquer une conception grecque ou occidentale sur des cultural performances (des manifestations culturelles spectaculaires) qui ne lui doivent rien". Le théâtre à l'ancienne reste alors un produit essentiellement esthétique, à une configuration relativement stable, et vouée à une répétition d'ellemême (presqu') à l'identique. Selon Pavis, la mise en scène de ce genre théâtral n'est $\mathrm{qu}^{\prime}$ "une des innombrables cultural performances", tandis que la notion de performance intègre "I'opposition esthétique/ non-esthétique"; "dans tous les cas, [...] une action est accomplie par la performance en question" (Pavis, 2007: 17). Une "action" ou, autrement dit, un événement qui se produit à mi-chemin entre la réalité telle quelle et sa mise en scène ou -ce qui revient au même- sa remise en question ad hoc. Une telle performance est plus ou moins "esthétique". Or, un spectacle en bonne et due forme "n'a pas un but seulement utilitaire ('performer' une cérémonie, un rituel)", mais en tout premier lieu, I'un "fictionnel et esthétique, en recherchant le beau" (Pavis, 2007: 17).

Si l'ère de la mise en scène commence au XIX ième siècle, avec le naturalisme et son effet du réel, une partie assez importante du spectacle contemporain brise l'illusion du "morceau de vie", sort du théâtre à l'ancienne et donne de plein pied dans le réel. Ce type de (re)présentation -performance, happening, etc.- renonce à faire semblant, et s'insère dans l'espace non-fictionnel qui l'entoure, voire même s'y confond. Cet ancrage dans l'immédiat est devenu presqu'une marque des créations artistiques actuelles. Le discours artistique et son sens se constituent aujourd'hui d'une manière éminemment contextuelle. Aussi peut-on dire qu'au moment du pragmatic turn en philosophie et dans les sciences du langage, les arts du spectacle empruntent eux aussi une voie pareille (l'une "pragmatique", en ce sens qu'on doit tout d'abord à Charles Morris): mettre en vedette le fonctionnement du signe dans sa relation avec les "utilisateurs", ou avec un "contexte" donné, d'où émergent un certain nombre de significations, profondément subjectivisées. Ce replacement du spectacle postmoderne dans son contexte de jeu témoigne d'un rapport assez insolite de l'art(éfact) au réel, d'une confusion préméditée à la Pirandello, des niveaux de la (re)présentation: la fiction s'insinue dans l'immédiat, tandis que le réel tourne en fiction. Pas une fois, le spectacle postmoderne amène son public dans la scène ou descend lui-mème dans la salle, dans la rue, dans des espaces non-conventionnels. Autrefois bien défendue par les poétiques classiques, la frontière entre la salle et la scène $s^{\prime}$ (entre)ouvre; tout le monde (ou presque) y est appelé à performer: le public, le DJ, l'ingénieur du son ou le maître des lumières, etc. L'ancienne structure fermée -création individuelle et d'habitude bien fignolée- se trouve maintenant remplacée par des formes ouvertes (happening, performance, installation, work in progress) qui n'aspirent plus au parachèvement du chef-d'oeuvre, mais à un tout autre type d'expression, dont l'enjeu reste à tirer au clair. Il en dérive bien des formes spectaculaires "hybrides", partagées entre théâtre et une sorte de reality-show, entre mise en scène et "mise en perf" ou 
"performise" (Pavis, 2007: 55), entre danse, théâtre et médias audiovisuels, entre présence (ou présentation) et représentation, entre performance, physical theatre (Thomas Leabhardt, apud Pavis, 2007) et "spectacle" en bonne et due forme, ce qui veut dire performance "théâtralisée" (Pavis, 2007). La représentation suppose un discours médiat, et généralement assumé comme fictionnel; tandis que la performance est plutôt non fictionnelle et subjective, et requiert une présence se manifestant i(-)mmédiatement, par un discours (verbalisé ou non) souvent autobiographique, qui conteste (ou seulement fait semblant de récuser) "I'idée de 're-présentation"' (Pavis, 2007: 14 -15). Ainsi, à partir des années '60, la performance comme spectacle vivant est une alternative à la mimesis du Stagirite. Ces expérimentations occupent actuellement une part assez importante des productions contemporaines, notamment underground, et parfois mainstream.

Dans la performance et les genres interactifs postmodernes, les rôles (du danseur, de l'acteur, du public) s'imbriquent pas une fois, et le spectacle débouche sur le contexte de la vie réelle qui l'entoure (l'espace du public, par exemple). Assez souvent, de telles performances reposent sur une poétique métafictionnelle et autoréférentielle, de la déconstruction et de la "dedoxification" (Hutcheon, 1997; Popa Blanariu, 2008, 2009, 2011, 2012). Cette poétique postmoderne s'attaque essentiellement aux codes esthétiques et culturels consacrés, tout comme aux moyens d'expression assujettis, et aux raisons d'être de tels langages. Cette remise en question des codes et langages artistiques (en tant qu'ensembles de conventions, contraintes et formes d'expression préétablies) s'associe fréquemment au penchant des artistes contemporains pour un style minimaliste et pour l'art conceptuel. La performance s'assume aussi une remise en question de l'esthétique par l'aisthesis: c'est-à-dire, remise en cause de l'aspect extérieur (visuel) de la (re)présentation, au profit de "I'introspection proprioceptive" (Suquet, 2009). Cela va de pair avec un rétrécissement de la "distance contemplative" (Suquet, 2009) d'entre le public et la scène, d'entre la vie et la fiction, d'entre le corps et son image culturelle. De telles performances sont plutôt live, "écrites" sur le vif, juste au moment où elles sont portées devant le public qu'elles sollicitent à fleur de peau. C'est dans ce "sens intérieur" du mouvement que dès 1912, Kandinsky a vu la matière et la fin de la danse à venir (Kandinsky apud Suquet, 2009). Prophétie en train de se confirmer?

Dans de telles circonstances, le public -professionnel ou pas- semble devoir s'adapter à un changement d'optique: d'une ancienne sémiologie de la mimesis (ou de la représentation), on avance petit à petit, depuis plusieurs décénnies, vers une pragmatique de la performance (ou présentation). En ce sens, Patrice Pavis (2007) remarquait à son tour le passage d'une "sémiologie de la mise en scène" à une "phénoménologie de la performance". Entre ces deux approches, il y a une différence concernant essentiellement, à mon avis, la manière de construire le spectacle et son sens. La construction d'un spectacle classique est généralement fermée, représentationnelle, c'est-à-dire bien 
définie par le dramaturge et le metteur en scène avant le début du spectacle. Dans ce cas, le spectacle reprend (ou re-présente) devant le public une forme artistique déjà bien établie et mise à l'épreuve pendant les répétitions. Ainsi conçu, le spectacle est une forme artistique relativement autonome et stable par rapport à la réaction immédiate du public et au contexte du jeu. Par contre, la performance et les genres interactifs postmodernes s'avèrent être essentiellement ouverts, fortement contextuels et redevables à la présence hic et nunc des artistes et du public. À la rigueur, la réaction et l'intervention du public dans l'espace du jeu peuvent changer le spectacle sur-le-champ; par conséquent, telle performance (ou présentation) peut radicalement différer d'une autre mise en oeuvre du même projet artistique. Tous ces changements, c'est la nature même de l'objet artistique postmoderne qui les imposent, tellement enraciné qu'il est dans son contexte (re)présentationnel. Celui-ci comporte toute une série de paramètres qui influent sur la production interactive du discours (verbal ou non verbal) et de son sens éminemment contextuel.

On pourrait dire que la performance et les genres interactifs comportent souvent l'aspect d'un spectacle -ou d'une "mise en perf"- de la communication. C'est-à-dire, non seulement les spectateurs regardent les artistes, mais en même temps, ceux-là se manifestent en tant que performers ad hoc. Cela veut dire que le public offre aux artistes (et aussi les spectateurs eux-mêmes s'offrent les uns aux autres) le spectacle de leurs propres réactions dans le contexte du jeu. À mon avis, cet aspect de la performance postmoderne rend d'autant plus légitime la question de la performativité dans l'interaction quotidienne, que soulève Goffman (1959; 1974): à quel point les comportements humains s'avèrentils avoir une dimension performative dans les circonstances habituelles de l'interaction des individus? II faut souligner que Goffman (1974) s'est intéressé de près aux traits communicatifs du comportement humain quotidien, dans ses analyses sociologiques ayant fait recours aux métaphores théâtrales, telles que: masque, rôle, personnage, décor, objet scénique.

Il faut aussi remarquer que dans des circonstances habituelles et aussi dans un contexte artistique, la dimension performative d'un fait ou d'un comportement peut être intentionnelle ou pas. En ce sens, John Cage, père fondateur de l'art performatif, était d'avis que l'intentionalité ne permet pas la libre manifestation de la créativité, cette découverte le conduisant à redéfinir le rôle et la manière spécifique d'agir de l'artiste: celui-ci -considérait Cage- doit seulement diriger l'attention du public vers ce qui existe déjà autour de soi, dans la nature ou dans le domaine de la technologie. Une performativité implicite, non intentionnelle réside donc dans des faits et phénomènes de tous les jours - les bruits de la pluie, la respiration, les klaxons dans la rue ou les appareils radio- dont Cage lui-même a fait l'expérience dans Imaginay Landscape N. ${ }^{\circ} .4$ (1951). De la même manière, certains comportements non intentionels du public et même des 
artistes peuvent, à mon avis, devenir performatifs et profondément significatifs dans le contexte de la performance postmoderrne.

Combinaison de sons voulue aléatoire, donc non-intentionnelle et nonreprésentationnelle, la musique de Cage trouve le plus souvent son sens grâce au décryptage subjectif du public et à sa disponibilité de participer au sémantisme de l'oeuvre. Comme Wagner avec son projet du "théâtre total", et Artaud auparavant, Cage a fait du point d'intersection de différents langages artistiques l'espace privilégié de la genèse du sens d'un oeuvre, chaque art tout en gardant son autonomie. Auteur de bien des performances, avec le chorégraphe Merce Cunningham, Cage a généralement composé une musique censée être performée, donc apte à collaborer avec d'autres langages artistiques à l'intérieur d'une même performance. Dans Points in Space (1986), Cage a composé la musique pour la chorégraphie de Cunningham, la musique et le mouvement étant pour la première fois associés lors de la première (Allain et Harvie, 2014). Conçues sans raport initial de "motivation" réciproque à la Saussure, la musique et la chorégraphie vont pourtant se compléter ainsi et s'illuminer l'une l'autre, dès qu'on les mettra ensemble, tout en proposant leur tandem à l'interprétation du public.

Avant Cage, Artaud saisit la même valence performative des composantes sonore et visuelle du spectacle: "les sons [y] interviennent comme des personnages", tandis que l'on "doit introduire dans la lumière un élément de ténuité, d'opacité en vue de produire le chaud, le froid, la colère, la peur etc." (Artaud, 1964: 146-147). Ce qu'Artaud désigne par "langage théâtral", c'est toujours une réalité multimodale, où s'imbrique une multitude de sous-langages: "langage auditif" $\mathrm{d}^{\prime}$ "incantation", "langage visuel des objets, des mouvements, des attitudes, des gestes", "langage de l'espace”, "langage des sons, des cris, des lumières, d'onomatopées" (Artaud, 1964: 138). Tout ce système de langages, "le théâtre se doit de l'organiser, en faisant avec les personnages et les objets de véritables hiéroglyphes, et en se servant de leur symbolisme et de leur correspondances, par rapport à tous les organes et sur tous les plans" (Artaud, 1964: 138). À tous ces niveaux, les composantes sensibles doivent devenir porteuses de significations dans la perspective de l'ensemble: il faut "prolonge[r] leur sens, leur physionomie, leurs assemblages jusqu'aux signes, en faisant de ces signes une manière d'alphabet" (Artaud, 1964: 138).

La découverte d'une couche "pré-expressive" dans le jeu de l'acteur est aussi centrale dans la poétique théâtrale d'Eugenio Barba. Le "pré-expressif" est non intentionnel, c'est l'énergie (ou "bios") produite avant toute intention d'expression consciente que l'acteur pourrait manifester conformément aux codes sociaux, culturels et artistiques qu'il s'est assumés. Ce contenu pré-intentionel et "pré-expressif"se laisse découvrir par les postures du performer, par l'espace qu'occupe son corps, par les processus physiques involontaires (tel le pouls) qui influent sur les possibilités d'expression et de communication de l'artiste (Barba et Savarese, 1991; Allain et Harvie, 2014). 


\section{LE SYMBOLISME EFFICACE. GESTE RITUEL, ACTION PERFORMATIVE, ACTE DE LANGAGE}

Le signe rituel est par excellence efficace. II n'a pas seulement une signification sémantique, mais aussi et surtout, I'une pragmatique. (On y renvoie au tryptique sémiotique de Morris, sémantique - syntaxe - pragmatique.) Tout acte rituel comporte absolument le sémantisme de l'efficace. On ne peut donc ignorer l'approche pragmasémantique du rite. On pense que c'est justement cela qui, sur un critère de I"'intentionalité collective", permet de dissocier le spectacle et le rituel, comme deux catégories distinctes de "faits sociaux" ou "institutionnels" (Searle 1998: 121 - 134). Le spectacle a une visée éminemment esthétique et le plus souvent, il se constitue comme une alternative fictionnelle du réel, tandis que le rituel (sacré notamment) est un scénario à caractère instrumental, un moyen d'agir sur le réel ou de s'en emparer, de le transformer à son gré ou de le garder tel quel. On cherche ainsi une réponse à une question de Patrice Pavis, qui porte toujours sur le rapport du jeu esthétique au jeu rituel, du homo religiosus à l'homo aestheticus:

L'opposition entre spectacle et action performative (rituel, cérémonie, jeu) se heurte à la difficulté de séparer l'esthétique et l'anthropologique. Est-il permis ou simplement pertinent de décrire, voire de se focaliser sur les effets esthétiques d'un rituel, ne passe-t-on pas à côté de l'essentiel? Si on se place, pour l'évaluer, sur le plan de la fiction, de la beauté des couleurs ou des formes, rend-on compte 'comme il faut' d'une cérémonie religieuse? (Pavis, 2007: 17).

Dans la "pensée sauvage" (Lévi-Strauss) et dans la pratique appropriée, le geste rituel est pourvu d'une valeur performative, qu'on peut désigner par le fameux adage austinien, "to do things with words". Cela revient à (vouloir/tenter/s'imaginer de) provoquer par le symbolisme corporel, tout comme par la parole, certains effets sur le monde. Pas une fois, l'énoncé kinésique est censé doué d'une valeur pareille à la force illocutoire du verbal (des exemples, il y en a apud Popa Blanariu, 2013b). On s'approprie ici le concept de performativité avec le sens que lui confère Searle (1998: 113-115; 133-134). La danse sacrée -remarque Greimas (1975: 94)- comporte l'intention de "transformation des contenus exprimés". On pense que cela revient à affirmer la dimension pragmatique du sémantisme de la chorégraphie rituelle. Plus précisément, par son attitude, par ses mouvements, par son costume et sa parure, l'acteur rituel qui "imite"le dieu "est"/"devient" effectivement, au cours de la cérémonie, le dieu qu'il est censé incarner (Cassirer, 1972: 61): "Le culte est le véritable outil de l'homme", car "malgré son cours régulier, la nature n'abandonne rien sans cérémonies". 
Inaugurée comme "trans(-)figuration" (changement de l'image apparente ou de la "figure", en vertu du rôle requis), la "transformation" du dans(act)eur vaut, en fin du compte, sa "transsubstantiation": une modification d'essence, pendant le rite (Popa Blanariu, 2008, 2013b, 2013c et 2015). À l'avis de Gadamer (2001: 91), I'acte de culte est, pour ses fidèles, toujours une "représentation autentique". Que veut-il dire par cela? Des Grecs anciens à Stanislavski, Artaud et Brecht, la représentation théâtrale revêt l'une des trois formes qu'a identifiées Gadamer (2001: 93-100): déguisement, métamorphose, changement. On peut dire que chez Artaud et Stanislavski, la raison d'être du théâtre est au juste la métamorphose de l'interprète et/ ou de son public: mutation "psychoémotionnelle"(Stanislavski) ou bien, "métaphysique","spirituelle”(Artaud). On peut même parler d'une poétique de la métamorphose chez Artaud et Stanislavski (Popa Blanariu, 2013c). Par contre, l'acteur brechtien -adepte du Verfremdungseffekt- subit un certain "changement" (alloiōsis), mais pas du tout une "métamorphose" (Popa Blanariu, 2013b, 2013c, 2015). Celle-ci a trait à l'essence du sujet; celle-là, à tel ou tel accident (Gadamer, 2001). Le dans(act)eur rituel est aussi le sujet d'une métamorphose à la Gadamer. Par cet effet pragmatique, du type performatif, le drame sacré (drômenon) diffère radicalement du déguisement ludique et/ ou esthétique. Le drômenon est un acte performatif, en ce sens que l'image rituelle "ne manifeste pas la chose, elle est effectivement cette choselà" (Cassirer, 1972: 60) ou le devient rituellement. Dans la "pensée sauvage", cela est possible grâce à une sorte de sorcellerie analogique qui repose sur l'identification du representamen (nom ou image) avec son designatum.

Lorsque les rites deviennent des"vestiges", la fonction descriptive survit à celle d'action effective (Lévi-Strauss, 1995: 410). Ce qui peut se "traduire" dans le métalangage d'Austin: le "constatif" va prévaloir sur le "performatif". L'expression -verbale ou non verbale- perd sa fonction rituelle, de "modification" du réel. Mais cela n'empêche point qu'elle n'en manifeste une autre, essentiellement esthétique (Popa Blanariu, 2008, 2013b, 2013c, 2013d et 2015). Les actes "illocutoires" que la danse rituelle peut accomplir recouvrent la taxonomie que Searle proposait à la suite d'Austin: directifs (inviter, demander, prier), promissifs, expressifs (remercier, féliciter, saluer). II y en a quelques exemples in Popa Blanariu (2013b). Les actes illocutoires accomplis au cours d'un spectacle sont, à l'opinion de Searle (1982: 113), des actes "réels" dans un univers de fiction. II faut ajouter que ceuxci sont tels du point de vue d'un public "observateur", qui reste à l'extérieur des faits fictionnels auquels il assiste. Par contre, pendant le rituel (ludus sacer), on réalise des actes réels dans un monde réel, du point de vue des "participants". (On garde ici la distinction, bien importante en anthropologie, entre ceux qui "participent" et ceux qui "observent": perspectives différentes, donc “lectures" différentes des faits.) 


\section{POUR FAIRE LE POINT}

Le XXì̀me siècle réinvente le corps et l'idée occidentale de spectacle. Une riche création chorégraphique et l'émergence d'une anthropologie du corps et des arts de la scène en témoignent. L'ancien spectacle respectueux de la Poétique du Stagirite, négocie son fief menacé par une performance protéiforme. Elle guette presque partout l'arrivée de son public: dans la salle, dans la rue ou dans des espaces (dits) non conventionels. Dans une certaine mesure, le spectacle s'échappe à l'académisme et va de pair avec la contreculture. Redevable aux paradigmes (post)modernes, la performance contemporaine repense le langage du corps et sa (re)mise en discours; autoréférentialité, intertextualité et interculturalité, critique socio-estetique n'y manquent pas.

Le corps parle toujours et inévitablement. Au début du XX ième siècle, la possibilité d'une grammaire du corps préoccupait constamment Edward Sapir. Au bout d'une longue recherche de la "Kinesics" -code universel des gestes-, Birdwhistell avouait son échec. En ce qui concerne la danse, on peut parler de code(s), mais il faut le faire prudemment, car la danse reste toujours partagée entre une sémiotique du signe et une (sémio-)pragmatique du discours. Il y a, sans doute, des postures pourvues d'une signification conventionnelle, mais la signification s'éclaircit, notamment dans la danse esthétique, grâce à l'ensemble de la construction chorégraphique. Seul l'emploi des éléments à signification (pré)déterminée ne peut produire un oeuvre d'art (Laban, 1994: 128). Surtout dans la danse esthétique (danse moderne ou ballet académique, par exemple), la dimension conventionnelle des signes -tant qu'elle existe- est nuancée par les paramètres discursifs de la mise en scène. En ce qui concerne la représentation magique et cultuelle, tant au niveau de l'expression qu'au rapport de l'expression au sens, il y prédomine des configurations kinésiques et posturales conventionnelles, qui envisagent des effets pragmatiques: soient-ils au niveau profane du monde ou à celui du rapport de l'homme au sacré (danses d'invocation, de supplication, de remerciement ou d'hommage des dieux, des ancêtres mythiques et du totem, du gibier, etc.). Rudolf Arnheim n'hésite pas à voir dans "I'art primitif" en général tout d'abord "un instrument pratique", à même "d'enregistrer et de transmettre des informations" et aussi d'exercer des "influences magiques" (Arnheim, 1979: 153).

L'histoire de la performance, de la (re)présentation et des arts du spectacle, dure depuis quelques millénaires déjà, Aujourd'hui encore, elle semble vouloir continuer. Comment? Cela reste à voir. Sous ce point d'interrogation, (presque) tous les arts contemporains se rassemblent, dans leurs efforts d'innover, et de survivre, à la fois. 


\section{RÉFÉRENCES BIBLIOGRAPHIQUES}

ALLAIN, P. et HARVIE, J. (2014). The Routledge Companion to Theatre and Performance. London: Taylor \& Francis.

ARNHEIM, R. (1954). Art and Visual Perception. A Psychology of the Creative Eye. Berkeley: University of California Press.

(1979). Arta şi percepția vizuală. O psihologie a văzului creator. Traduction par F. Ionescu, Bucureşti: Meridiane.

ARTAUD, A. (1964). Le théâtre et son double suivi de Le théâtre de Séraphin. Paris: Galimard.

AUSTIN, J.L. (1970). Quand dire, c'est faire. Traduction par G. Lanne. Paris: Seuil.

AUGUSTIN (2002). De doctrina christiana: introducere în exegeza biblică. Édition bilingue, établissement du texte latin et traduction roumaine par Marian Ciucă. Introduction, notes et bibliographie par Lucia Wald. Bucureşti: Humanitas.

BARBA, E. et SAVARESE, N. (1991). A Dictionary of Theatre Anthropology: The Secret Art of the Performer. London: Routledge.

BATESON, G. et RUESCH, J. (1951). Communication: The Social Matrix of Psychiatry. New York: Norton.

(1988). Communication et société. Paris: Seuil.

BENVENISTE, É. (1966-1974). Problèmes de linguistique générale, t. I - II. Paris: Gallimard.

(2000). Probleme de lingvistică generală. Traduction par L. M. Dumitru. Bucureşti: Teora.

BOURCIER, P. (1994). Histoire de la danse, t. I. Paris: Seuil.

BOUVET, D. (1997). Le Corps et la métaphore dans les langues gestuelles. Paris: L'Harmattan.

CASSIRER, E. (1972). Philosophie des formes symboliques (t. II). Traduction française de l'allemand par J. Lacoste. Paris: Minuit.

CORBIN, A., COURTINE, J.-J. et VIGARELLO, G. (2006). Histoire du corps. Tome 3: Les mutations du regard. Le XX-ième. Paris: Seuil.

DELAVAUD-ROUX, M.-H. (1993). Les danses armées en Grèce antique. Aix-en-Provence: Université de Provence.

DURKHEIM, É. (1968). Les Formes élémentaires de la vie religieuses. Le Système totémique en Australie. Paris: PUF.

(1995). Formele elementare ale vieții religioase. Traduction par Magda Jeanrenaud et Silviu Lupescu. Iaşi: Polirom.

ECO, U. (1975). Trattato di semiotica generale. Milano: Bompiani.

(1982). Tratat de semiotică generală. Traduction par A. Giurescu et C. Radu. Bucureşti: Editura Ştiințifică şi Enciclopedică .

(1988). Sémiotique et philosophie du langage. Paris: PUF.

ELIADE, M. (1976). Histoire des croyances et des idées religieuses. Paris: Payot. 
(2000). Istoria credințelor şi ideilor religioase. Traduction et postface de Cezar Baltag. Bucureşti: Univers Enciclopedic.

GADAMER, H.-G. (1996). Vérité et méthode. Paris: Seuil.

(2001). Adevăr şi metodă. Traduction par Gabriel Cercel, Larisa Dumitru, Gabriel Kohn,

Călin Petcana. Bucureşti:Teora.

GENETTE, G. (1991). Fiction et diction. Paris: Seuil.

GOFFMAN, E. (1959). The Presentation of Self in Everyday Life. New York: Doubleday. (1974). Les Rites d'interaction. Paris: Minuit.

(2001). Mise en scène de la vie quotidienne, t. 1-2. Paris: Minuit.

GREIMAS, A. J. (1970). Du sens. Paris: Seuil.

(1975). Despre sens. Traduction par Maria Carpov. Bucureşti: Univers.

HALL, E.T. (1971). La dimension cachée. Paris: Seuil.

(1973). Le Langage silencieux. Paris: Mame.

HELBO, A. (sous la direction de) (2011). Performance et savoirs. Bruxelles:De Boeck.

HUTCHEON, L. (1989). The Politics of Postmodernism. London \& New York: Routledge.

(1997). Politica postmodernismului. Traduit par Mircea Deac. Bucureşti: Univers.

JAKOBSON, R. (1963). “Linguistique et poétique”. En Essais de linguistique générale. Paris: Minuit.

LABAN, R. (1994). La Maîtrise du mouvement. Traduit par Jacqueline Challet-Haas et Marion Bastien. Paris: Actes Sud.

LEROI-GOURHAN, A. (1964-1965). Le geste et la parole, t. I-II. Paris: Albin Michel. (1983). Gestul şi cuvântul. Traduction par M. Berza. Bucureşti: Meridiane.

LÉVI-STRAUSS, C. (1995). Mitologice. Traduction par loan Pânzaru. Bucureşti: Babel. (2009). Mythologiques (I). Le cru et le cuit. Paris: Plon.

MARCUS, S. (sous la direction de et avec une étude introductive par) (1982). Semiotica matematică a artelor vizuale. Bucureşti: Editura ştiințifică şi enciclopedică.

MORRIS, C. (1971). Writings on the General Theory of Signs. The Hague: Mouton.

PAVIS, P. (2002). Dictionnaire du théâtre. Préface de Anne Ubersfeld, édition revue et corrigée. Paris: Armand Colin.

(2007). La Mise en scène contemporaine. Origines, tendances, perspectives. Paris: Armand Colin.

(2012). Dicționar de teatru. Traduction par Nicoleta Popa Blanariu et Florinela Floria. laşi: Fides.

PEIRCE, C. S. (1978). Écrits sur le signe. Rassemblés, traduits et commentés par Gérard Deledalle. Paris: Seuil.

POPA BLANARIU, N. (2008). Când gestul rupe tăcerea. Dansul şi paradigmele comunicării [Quand le geste rompt le silence. La danse et les paradigmes de la communication]. Iaşi: Fides.

(2009). "Aşa e, dacă vi se pare... Limbajul corpului şi discursul postmodern. Dans contemporan în România, 2006-2009". Analele Universității "Ştefan cel Mare" din Suceava, seria 


\section{NICOLETA POPA BLANARIU}

Filologie. B. Literatură XV.1 ("Literatura postmodernă"), 195-209 (http://www.analelitere.usv.ro [06/09/2016]).

(2011). "(Re)prezentarea. Narcis şi postmodernii". Vitraliu 36 (1-2), 26-27. (2012). "O cameră, un labirint, un jurnal şi trei rochii". Vitraliu 38 (1-2), 46-47.

(2013a). "Towards a Framework of a Semiotics of Dance." CLCWeb: Comparative Literature and Culture 15.1 (http://dx.doi.org/10.7771/1481-4374.2183 [06/09/2016]).

(2013b). "Performativity and Ritualistic Interaction". En Perspectives on Interaction, E. Bonta (ed.), 35-46. Newcastle: Cambridge Scholars Publishing.

(2013c). "Towards a Poetics of Performance: Embodiment and 'Verfremdungseffekt' as Fuzzy Aspects of Acting". The International Journal of the Humanities: Annual Review 10, 79 91. (http://ijhar.cgpublisher.com/product/pub.281/prod.15 [06/09/2016]).

(2013d). “Theatricalization of Ritualistic Gesture and Dancing. A Semiotic Approach”. En A Semiotic Reader, Geoffrey Sykes (ed.), 183-209. Stanwell Tops Australia: Southern Semiotic Review.

(2013e). "Narrativity as Transmediality. Dancing Literature: a Reverse Ekphrasis". Journal of Comparative Literature and Aesthetics (special issue: On Intermedial Aesthetics and World Literatures) XXXVI (1-2), 129-141, Asunción López Varela (Guest Editor) (https://www.ucm.es/ siim/journal-of-comparative-literature-and-aesthetics [06/09/2016]).

(2014a). "Semiotic and Rhetorical Patterns in Dance and Gestural Languages". Southern Semiotic Review 4 (http://www.southernsemioticreview.net/[06/09/2016]). (2014b). "À partir de Greimas. Semiotic and Communication Patterns in Dance". Southern Semiotic Review 3 (http://www.southernsemioticreview.net [06/09/2016]).

(2015). "Paradigms of Communication in Performance and Dance Studies". CLCWeb: Comparative Literature and Culture 17.2 (http://dx.doi.org/10.7771/1481-4374.2331 [06/09/2016]).

(2016). Când literatura comparată pretinde că se destramă. Studii şi eseuri, vol. II: (Inter)text şi (meta)spectacol [Quand la littérature comparée prétend s'évanouir, vol. II: (Inter)texte et métaspectacle]. Bucureşti: Eikon.

SAMOSATE, L. de (2009). "Of Pantomime ". En The Works of Lucian of Samosata. Translated by H. W. Fowler and F. G. Fowler: http://ebooks.adelaide.edu.au/l/lucian/works/chapter31.html [06/09/2016].

(1983). Opere alese. Traduction par R. Hâncu, deuxième édition. Bucureşti: Univers.

SAPIR, E. (1967). "L'influence des modèles inconscients sur le comportement social". En Anthropologie. Paris: Minuit.

SAUSSURE, F. de (1997). Cours de linguistique générale. Paris: Payot.

(1998). Curs de lingvistică generală. Traduction par I. Izverna-Tarabac. Iaşi: Polirom.

SEARLE, J. R. (1982). "Le statut logique du discours de la fiction". En Sens et expresion: études de théorie des actes de langage. Paris: Minuit. 
(1998). Mind, language and Society. Philosophy in the Real World. New York: Basic Books.

SCHECHNER, R. (2002). Performance Studies. London: Routledge.

SUQUET, A. (2009). "Scene. Corpul dansând: un laborator al percepției". En Alain Corbin, Jean-

Jacques Courtine, et Georges Vigarello, Istoria corpului, vol. 3. Traduction par Simona

Mihalache, Mihaela Arnat, Muguraş Constantinescu, Giuliano Sfichi, 455-504. Bucureşti: Art. TARASTI, E. (1996). Sémiotique musicale. Limoges: PULIM.

WINKIN, Y. (2000). La Nouvelle Communication. Nouvelle édition. Paris: Seuil.

(2001). Anthropologie de la communication. De la théorie au terrain. Nouvelle édition.

Paris: Seuil.

Recibido el 12 de enero de 2016.

Aceptado el 1 de noviembre de 2016. 
